\title{
An Efficient Radio Map Updating Algorithm based on K-Means and Gaussian Process Regression
}

\author{
Jianli Zhao ${ }^{1}$, Xiang Gao ${ }^{1}$, Xin Wang ${ }^{1,3}$, Chunxiu $\mathrm{Li}^{1}$, Min Song ${ }^{2}$ and \\ Qiuxia $\operatorname{Sun}^{4}$ \\ ${ }^{1}$ (College of Computer Science and Engineering, Shandong University of Science and \\ Technology, Tsingtao, China) \\ ${ }^{2}$ (College of Engineering, Michigan Technological University, Michigan, USA) \\ ${ }^{3}$ (Beijing Key Laboratory on Integration and Analysis of Large-scale Stream Data, \\ Beijing China) \\ ${ }^{4}$ (College of Mathematics and Systems Science, Shandong University of Science and \\ Technology, Tsingtao, China) \\ (E-mail: zhaojianli@gmail.com)
}

\begin{abstract}
Fingerprint-based indoor localisation suffers from influences such as fingerprint pre-collection, environment changes and expending a lot of manpower and time to update the radio map. To solve the problem, we propose an efficient radio map updating algorithm based on K-Means and Gaussian Process Regression (KMGPR). The algorithm builds a Gaussian Process Regression (GPR) predictive model based on a Gaussian mean function and realises the update of the radio map using K-Means. We have conducted experiments to evaluate the performance of the proposed algorithm and results show that GPR using the Gaussian mean function improves localisation accuracy by about $13.76 \%$ compared with other functions and KMGPR can reduce the computational complexity by about $7 \%$ to $20 \%$ with no obvious effects on accuracy.
\end{abstract}

\section{KE YW ORDS}
1. Indoor localisation.
2. Radio Map updating.
3. GPR.
4. Machine learning.

Submitted: 8 October 2016. Accepted: 14 March 2018. First published online: 30 April 2018.

1. INTRODUCTION. With the rapid development of mobile devices and technologies, location-aware applications have become more and more popular. The Global Positioning System (GPS) is known as one of the most effective systems for providing positions for 
outdoor applications. However, in high-density urban areas and indoor scenes, it is difficult to provide accurate position because of the attenuation and interference of signals. Therefore, indoor positioning technology with high accuracy has become a challenging research problem (Park et al., 2010). In recent years, WiFi-based positioning techniques have received considerable attention because of their wide application. A method is proposed using the collection of a dense dataset of WiFi fingerprints and their associated true locations to build a "radio map" (Mahtab Hossain et al., 2013). Yu and Dutkiewicz (2013) and Mihaylova et al. (2011) have investigated WiFi multipath distortion and noise to improve localisation accuracy and robustness. Some researchers (Au et al., 2013; Jun et al., 2013; Seitz et al., 2010) have implemented collaborative localisation by combining WiFi and Pedestrian Dead Reckoning. Ferris et al. (2007) proposed WiFi-based simultaneous localisation and mapping through related robotics.

Most previous positioning methods employ Received Signal Strength (RSS) as a measurement for position determination. For most off-the-shelf devices, such as WiFi or ZigBee devices, RSS fingerprints are easily available. In these methods, localisation is divided into two phases: training and localisation. The training phase builds a radio map offline, the radio map records RSS received from nearby WiFi Access Points (APs) and the Reference Points' (RPs) positions where the RSS are collected. In the localisation phase, when users send a location query with the current RSS fingerprint, the localisation algorithm retrieves the radio map to estimate the corresponding location.

The biggest challenge of WiFi-based indoor localisation is timely updating of the radio map as things change. The propagation of wireless signals is easily affected by surrounding environmental changes, such as architectural change, change of interior decoration, and instantaneous interference (Kaemarungsi and Krishnamurthy, 2012). These changes may cause the radio map to become outdated but re-building the radio map is costly and laborious (Chintalapudi et al., 2010). In this paper, we apply Gaussian Process Regression (GPR) combined with K-Means to update a global radio map using only one part of the Received Signal Strength Indications (RSSI) to reduce the workload and computational complexity of GPR.

GPR is a non-parametric modelling method, and it is suitable for modelling the received signal strength (Rasmussen and Williams, 2005). The advantage of GPR for modelling WiFi fingerprints is that it includes not only the mean estimation of received signal strength, but also the variance. The mean and variance can be used to reflect the characteristics of the received signal strength even though this may disregard some potentially useful information. However, GPR can require extensive computational resources and may not be suitable for real-time localisation. So, we propose an efficient radio map updating algorithm based on K-Means and GPR. Our experiment shows that the proposed algorithm can greatly reduce the computational complexity and system overhead without sacrificing localisation accuracy.

The contributions of this paper are two-fold. Firstly, a Gaussian mean function-based GPR model is applied for WiFi localisation and compared with other models. Secondly, we propose an efficient algorithm of updating the radio map based on K-Means and GPR. We are able to update the radio map online with a lower computational burden and faster than before.

The rest of the paper is organised as follows. In Section 2, related work is discussed. The details of the proposed algorithm are presented in Section 3. The experimental results are presented in Section 4, and Section 5 provides conclusions. 
2. RELATED WORK. Recently, many approaches have been proposed to solve the problem of effectively updating the radio map. Traditional methods including triangulation interpolation, linear interpolation and Kriging interpolation were proposed by Chai and Yang (2007). However, when fingerprints are sparse, these methods have difficulties in forecasting the RSSI and thus result in poor localisation accuracy. Therefore, other methods have been proposed, including free-calibration methods (Wu et al., 2013; Kim et al., 2012), crowdsourcing methods (Yang et al., 2013; Rai et al., 2012; Chang and Han, 2014; Wu et al., 2015a; 2015b; Hansen et al., 2010), machine learning models (Pan et al., 2007; Ferris et al., 2006; Bekkali et al., 2011; Richter and Toledano-Ayala, 2015), and the combination of previous methods introduced by Atia et al. (2013).

Wu et al. (2013) proposed Wireless Indoor Localisation (WILL) to record RSSI vectors and the relative distance between them from the embedded sensors to build the radio map. The limitation of WILL is that the quality of fingerprint largely relies on the accuracy of a step counter. Another method introduced by Yang et al. (2013) can deal with an unstable signal and construct a metropolitan-cell-based radio map efficiently. However, the proposed interpolation technology is not accurate since it only relies on the relative distance.

The second method is the utilisation of crowdsourcing. The radio map is updated with the most recently measured RSS and inertial sensors' data uploaded by users. However, designing a sustainable incentive mechanism for crowdsourcing remains a challenge. Chang et al. (2014) used various inertial sensors to collect the users' path data and update the radio map by interpolation. However, it has limitations in labelling the fingerprints which is rooted in incorrect prediction of a user's path, inaccurate step count and heading. $\mathrm{Wu}$ et al. (2015a) mapped the fingerprint space to the floor plan in a stress-free form, which results in fingerprints labelled with physical locations. It also relies on inertial sensors' data.

The third method is based on machine learning. Machine learning algorithms are applied in many fields, such as recommender systems, and so on (Wu et al., 2017; Ji et al., 2017). Pan et al. (2007) proposed transferring learning to deal with the signal change. This method can learn the correlation between the locations and fingerprints. However, offline training and online localisation have a high computational cost. A Gaussian process was used to predict Global System for Mobile Communications (GSM) and WiFi signal strength at earlier stages, which adds an offset on the basis of a zero-mean function to solve the limitations (Ferris et al., 2006). This is limited in practical application because the locations of APs are required. Bekkali et al. (2011) applied a Gaussian process to indoor localisation but did not propose the usage of a hyper-parameter optimisation method and a mean function. The GPR model has been used for predicting Wireless Local Area Networks (WLAN) fingerprints based on mean and covariance functions (Richter and Toledano-Ayala, 2015). Experimental results show that a constant mean function has the best performance, but these mean functions are not in line with the RSS, and do not take into account the impact of reference points' density on RSS modelling.

Atia et al. (2013) applied a Gaussian process to online-calibrated radio maps for indoor localisation. The gradient descent algorithm was used for hyper-parameter optimisation, and a path loss mean model was proposed. However, sparse deployment of APs may degrade the performance and the APs' locations are needed. Liu et al. (2016) focused on a GPRP method based on GPR and the Naive Bayes algorithm to reduce the computational complexity of indoor localisation, and it retains the flexibility of the GPR models. However, the environment is limited, and it requires additional hardware. Kumar et al. (2016) applied 
GPR which considered the variance of input for indoor localisation. It employed principal component analysis to choose the stronger WiFi AP. However, localisation accuracy is achieved at the expense of $\mathrm{O}\left(n^{3}\right)$ computational complexity.

Our study is motivated by these former works, but we solve the problem from a different approach and mainly focus on improving the updating efficiency online without sacrificing localisation accuracy. We propose a novel algorithm to update the radio map by K-Means GPR (KMGPR). We first build a GPR predictive model based on a Gaussian Mean GM) function and then an efficient radio map updating algorithm KMGPR based on K-Means and GPR is applied to reduce the computational complexity of GPR.

\section{THE ALGORITHM OF RADIO MAP UPDATING BASED ON K-MEANS AND GAUSSIAN PROCESS REGRESSION.}

3.1. GPR in fingerprint-based localisation. GPR is a non-parametric and probabilistic approach to regression. It is a probability distribution over functions determined by a mean function $m(x)$ and covariance function $k\left(x: x^{\prime}\right)$. Fingerprint-based localisation is used to predict the location based on the RSSI, and we can use GPR to predict the current RSSI. Table 1 introduces the terms used in this work.

Here, given the training data $X$ and $Y$, we could predict the posterior distribution of a function at an arbitrary point $x_{*}$. The posterior distribution of function values is a Gaussian distribution with $\mu_{x_{*}}$ and $\sigma_{x_{*}}^{2}$ :

$$
\begin{gathered}
p\left(f \mid x_{*}, X, Y, \theta\right) \sim N\left(\mu_{x_{*}}, \sigma_{x_{*}}^{2}\right) \\
\mu_{x_{*}}=K_{*}\left(K+\sigma_{n}^{2} I\right)^{-1} Y \\
\sigma_{x_{*}}^{2}=K_{* *}-K_{*}^{T}\left(K+\sigma_{n}^{2} I\right)^{-1} K_{*}+\sigma_{n}^{2} I
\end{gathered}
$$

The covariance matrix between the $n$ reference points is given in Equation (4).

$$
K=\left|\begin{array}{cccc}
k_{11} & k_{12} & \cdots & k_{1 n} \\
k_{21} & k_{22} & \cdots & k_{2 n} \\
\vdots & \vdots & & \vdots \\
k_{n 1} & k_{n 2} & \cdots & k_{n n}
\end{array}\right|
$$

In fingerprint-based indoor localisation, GPR is used to construct the likelihood model based on WiFi signal strength. The key part is in Equations (2) and (3), which represent the distribution of the RSS prediction.

Fortunately, we can learn and optimise the parameter through maximising the log likelihood function of the observation $Y$ given by

$$
\log p(Y \mid X, \theta)=-\frac{1}{2} Y^{T}\left(K+\sigma_{n}^{2} I\right)^{-1} Y-\frac{1}{2} \log \left|K+\sigma_{n}^{2} I\right|-\frac{n}{2} \log 2 \pi
$$

We optimise Equation (5) by conjugating gradient descent and computing the partial derivatives of the log likelihood.

$$
\frac{\partial}{\partial \theta_{j}} \log p(Y \mid X, \theta)=\frac{1}{2} \operatorname{tr}\left(\left(K^{-1} Y\right)\left(K^{-1} Y\right)^{T} \frac{\partial K}{\partial \theta_{j}}\right)
$$

The most complex step of the calculation is the hyper-parameter optimisation in Equation (6). It needs to recalculate the inversion of the covariance matrix $K$ for each 
Table 1. Table of GPR parameter symbols in fingerprint-based localisation.

\begin{tabular}{ll}
\hline Symbol & Definition \\
\hline$X$ & $\begin{array}{l}\text { a set of coordinates: } X=\left\{x_{i}, i=1, \ldots, n\right\} \\
\text { the collected RSS data of } X: Y=\left\{y_{i}, i=1, \ldots n\right\}\end{array}$ \\
$y_{i}$ & $y_{i}=\left\{A P_{j}, j=1, \cdots M\right\}$ is the set of RSS data of $x_{i}$ \\
$\mu_{x_{*}}$ & the mean of Gaussian distribution \\
$\sigma_{x_{*}}^{2}$ & the variance of Gaussian distribution \\
$x_{*}$ & the predicted points $\mathrm{x}_{*}=\left\{x_{i}^{*}, i=1, \cdots M\right\}$ \\
$K$ & the $n \times n$ covariance matrix of $X$ \\
$K_{*}$ & the $n \times 1$ vector of covariance between the $X$ and $x_{*}$ \\
$K_{* *}$ & the covariance matrix of $x_{*}$ \\
$\sigma_{n}$ & the Gaussian noise variance \\
$\sigma_{f}$ & the signal variance \\
$l$ & the length scale of the covariance function \\
$\theta$ & the hyper-parameters and $\theta=\left\{\sigma_{n}^{2}, \sigma_{j}^{2}, l\right\}$ \\
\hline
\end{tabular}

new $\theta$, which costs time $O\left(n^{3}\right)$, where $n$ is the number of reference points that collect the WiFi fingerprints. Therefore, the method to reduce the computational complexity requires an effective gradient descent algorithm or other efficient machine learning methods.

3.2. GPR modelling using Gaussian mean function. A Gaussian process is normally a zero-mean prior distribution, and most of the predicted RSS values tend to be zero when lacking training data in a large positioning region. This will result in a higher localisation error. To find a more suitable GPR prior model to predict RSS, a variety of mean functions were evaluated. We used a GPR predictive model based on a Gaussian Mean (GM) function and Equation (2) is displaced by Equations (7) and (8). Moreover, we also compare three other different mean functions: the zero-mean function, the linear mean function and the constant mean function.

$$
\begin{gathered}
\mu_{x_{*}}=m\left(x_{*}\right)+K_{*}\left(K+\sigma_{n}^{2} I\right)^{-1}(Y-\mu(x)) \\
m\left(x_{*}\right)=p\left(f \mid x_{*}, X, Y, \theta\right)
\end{gathered}
$$

When using a GM function to predict RSS, it can effectively capture the RSS feature at a reference point compared with the constant and linear mean functions. This is in line with the characteristics of the signal fluctuations and is conducive to RSS modelling.

3.3. Online radio map updating algorithm. GPR consumes more computational effort when updating the radio map using Equations (3) and (7). So, it is not conducive to indoor real-time localisation. To overcome this problem, we propose an efficient algorithm of radio map updating based on K-Means and Gaussian Process Regression. The KMGPR algorithm can effectively reduce the computational complexity of the GPR predictive model.

This algorithm utilises K-Means clustering on the initial radio map. All the reference points $\left\{x_{i}\right\}_{i=1}^{n}$ in the target area were divided into several clusters based on the Euclidean distance of locations. In every cluster, we use GPR to predict other fingerprints to update the original radio map. When building the GPR model, we only consider the training data that belongs to the same cluster, so it is very efficient. The KMGPR algorithm can be summarised as the pseudo-code in Table 2.

As mentioned above, reducing the GPR computational complexity is an important goal of our work because GPR requires a high computational load. We use clustering to solve 
Table 2. The KMGPR algorithm.

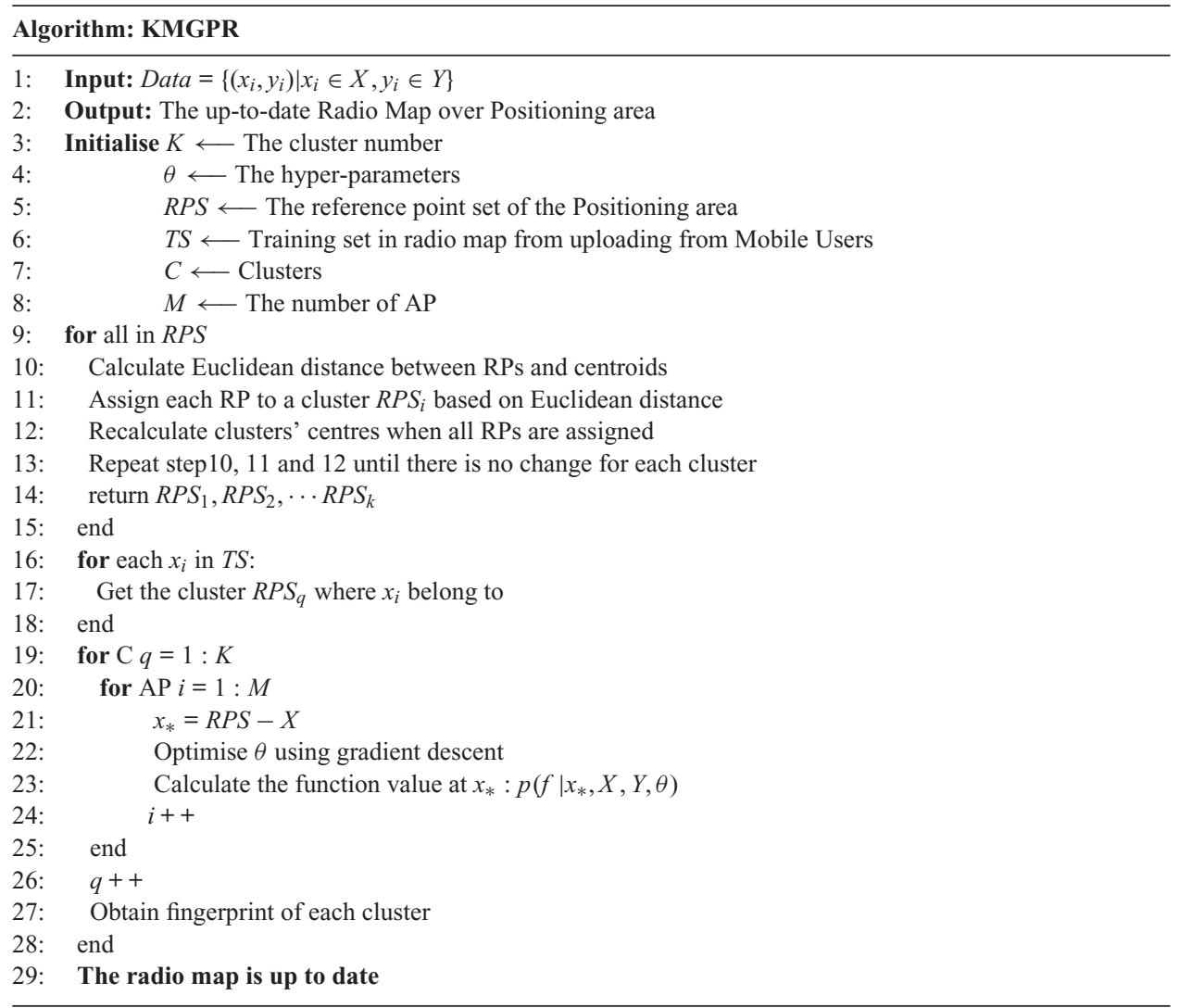

the problem. The reference point set of the entire area can be divided into separate sets by K-Means based on the correlation of locations.

When the number of reference points in a cluster is $m$, the KMGPR algorithm simplifies Equations (2) and (3) by only considering $m$ reference points. Therefore, $K_{*}$ and $Y$ reduce to a $m \times 1$ vector, and $\operatorname{cov}(Y)$ in Equation (2) to a $m \times m$ matrix. So, when updating the radio map online, $K$ clusters can conduct GPR prediction concurrently. Compared to the complexity $O\left(n^{3}\right)$ when considering all $n$ reference points in the region, in the KMGPR, we also use the $\mathrm{n}$ reference points and they are divided into $\mathrm{K}$ clusters, the complexity is $O\left(\mathrm{~m}^{3}\right)$ for a cluster and the total complexity is $K^{*} O\left(\mathrm{~m}^{3}\right)$ for all $\mathrm{K}$ clusters. In general, $K^{*} O\left(m^{3}\right)<<O\left(n^{3}\right)$, because $m<n$.

4. SIMULATION AND EXPERIMENT. We conducted the simulation and experimental study in two scenarios. In the first scenario, we used the experimental data set introduced by Laoudias et al. (2013), which is a typical office environment with an area of $560 \mathrm{~m}^{2}$ (see Figure 1). In the second scenario, we set up our testbed in a real environment.

\subsection{Simulations.}

4.1.1. Simulations setup. There are nine APs in the first scenario. The experimental data set contains RSS from all available APs at 105 distinct reference points and 


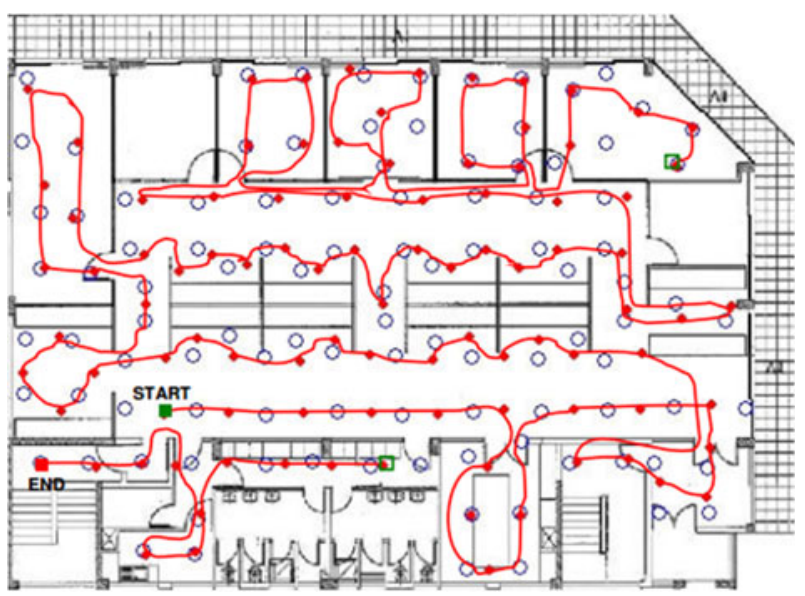

Figure 1. Experimental area introduced by Laoudias et al. (2013) (Scenario 1).

corresponding true locations for comparison. The data set was collected from a Samsung Nexus S containing training and test data. The training data has 2,100 fingerprints for 105 reference points; 20 fingerprints at each reference point. The researcher collected additional test data by walking along a predefined path. The path consists of 96 locations, most of which do not coincide with the reference points. As shown in Figure 1, there are 105 blue circles representing the reference points and the red curve connects 96 red points as the test path.

4.1.2. GPR model evaluation. With a GPR model, we use just a part of the reference point set to update the whole radio map and the final fingerprint density is the same. Different numbers of reference points were randomly selected as training points to update the whole 105 reference points: 20, 40,60,80 and 100 and they were not necessarily evenly distributed across the entire indoor area. In order to avoid errors caused by randomness, we conducted 10-fold cross-validation and obtained the mean error as the final result in the RSS estimation and localisation test. To illustrate the quality of GM in updating the RSS radio map, we also compared three other mean functions: (1) the Zero-mean function (Zero), as $m(x)=0$; (2) the Constant mean function (Const), as $m(x)=c$ and $c$ is a constant; (3) the Linear mean function (Lin), as $m(x)=a^{T} x$ and $a$ is a linear coefficient vector.

4.1.2.1. RSS estimation accuracy. In the simulation we used 10 -fold cross-validation to determine the quality of the models, and the test error and the Root Mean Squared Error (RMSE) of different models were investigated. Test error is defined as follows:

$$
\operatorname{Res}_{i}=F_{i}-\bar{F}_{i}
$$

where $F_{i}$ and $\bar{F}_{i}$ are estimated RSS values and true RSS values at reference point $i$ respectively. We also computed the RMSE over the remaining reference points which were not training points among the 105 reference points; when the number of reference points is $m$, the RMSE is as defined in Equation (10).

$$
R M S E=\sqrt{\sum_{i=1}^{m} \operatorname{Res}_{i}^{2} / m}
$$


Table 3. Test error and RMSE of the GPR model for different mean functions.

\begin{tabular}{|c|c|c|c|c|c|c|c|c|}
\hline \multirow{2}{*}{$\begin{array}{l}\text { Mean Function/ } \\
\text { The number } \\
\text { of RPs }\end{array}$} & \multicolumn{4}{|c|}{ Test Error/dBm } & \multicolumn{4}{|c|}{$\mathrm{RMSE} / \mathrm{dBm}$} \\
\hline & Zero & Const & Lin & GM & Zero & Const & Lin & GM \\
\hline 20 & 6.458 & $5 \cdot 932$ & $6 \cdot 413$ & $5 \cdot 275$ & $6 \cdot 752$ & $6 \cdot 01$ & 6.481 & $5 \cdot 318$ \\
\hline 40 & $4 \cdot 219$ & $4 \cdot 225$ & $5 \cdot 747$ & $4 \cdot 033$ & $5 \cdot 849$ & $5 \cdot 519$ & $6 \cdot 331$ & 4.064 \\
\hline 60 & 4.069 & $3 \cdot 644$ & $5 \cdot 271$ & $3 \cdot 392$ & 4.805 & $5 \cdot 12$ & $6 \cdot 276$ & $3 \cdot 562$ \\
\hline 80 & $4 \cdot 004$ & $3 \cdot 592$ & $4 \cdot 745$ & $3 \cdot 169$ & 4.704 & $5 \cdot 078$ & $6 \cdot 135$ & $3 \cdot 376$ \\
\hline 100 & 3.749 & $3 \cdot 547$ & $4 \cdot 288$ & $2 \cdot 869$ & 4.508 & $4 \cdot 806$ & $5 \cdot 876$ & $3 \cdot 142$ \\
\hline
\end{tabular}

Table 4. Average localisation error for different mean functions.

\begin{tabular}{|c|c|c|c|c|}
\hline \multirow{2}{*}{$\begin{array}{l}\text { The number } \\
\text { of RPs }\end{array}$} & \multicolumn{4}{|c|}{ Localisation error/m } \\
\hline & Zero & Const & Lin & GM \\
\hline 20 & $3 \cdot 523$ & 2.949 & $3 \cdot 334$ & 2.898 \\
\hline 40 & $2 \cdot 764$ & $2 \cdot 618$ & 3.073 & 2.562 \\
\hline 60 & $2 \cdot 607$ & $2 \cdot 553$ & $2 \cdot 748$ & $2 \cdot 214$ \\
\hline 80 & $2 \cdot 482$ & $2 \cdot 389$ & 2.699 & $2 \cdot 102$ \\
\hline 100 & $2 \cdot 475$ & $2 \cdot 376$ & 2.639 & 2.078 \\
\hline
\end{tabular}

Table 3 shows the test error and RMSE of the GPR model for different mean functions. The analysis of the test error and RMSE showed similar experimental results when different numbers of reference points were selected as the training data set. GM consistently outperformed the constant mean function, zero mean function and linear mean function. Compared with other mean functions, the test error and RMSE of GM were $4.41 \sim 35.65 \%$ and $11.51 \sim 46.53 \%$ lower, respectively.

4.1.2.2. Localisation accuracy estimation. In order to further verify the model performance of GPR based on GM, we present the results of the localisation accuracy of the 96 test points using Weighted K-Nearest Neighbour (WKNN). WKNN is a popular algorithm for fingerprint-based indoor localisation, which employs K nearest RPs by Euclidean distance to estimate a user's location. The weight is obtained from the calculated distance and $\mathrm{K}$ is the number of RPs whose distance is less than a set threshold. For experimental comparison, the training data of all 105 reference points were used for the construction of the Original radio map (ORI). The Localisation Error is defined as follows:

$$
d=\sqrt{(x-\hat{x})^{2}+(y-\hat{y})^{2}}
$$

where $(x, y)$ and $(\hat{x}, \hat{y})$ are estimated location and true location, respectively. Clearly, the smaller the Localisation Error, the better the algorithm performs.

Table 4 lists the average localisation accuracy for different GPR models after 10-fold cross-validation. For different training sets of RPs, the most accurate GPR model is the one with the GM function. When compared to other models, the localisation accuracy of GM has improved by an average of $13.76 \%$. When the number of training reference points is 60 , the localisation accuracy can be improved by $13.27 \sim 19.43 \%$. In Figure 2(a), the CDF of localisation error for different mean functions is shown. The probability of GM's localisation accuracy is a little lower than the ORI, but higher than the other three models. 
(a)

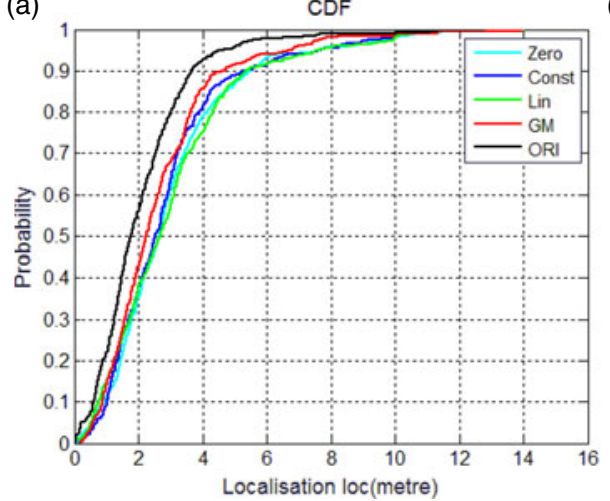

(b)

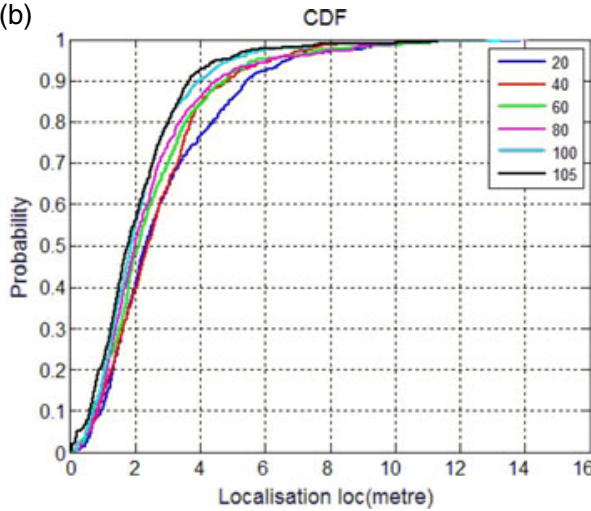

Figure 2. The Cumulative Distribution Function (CDF) of localisation error. (a) different mean functions; (b) different numbers of RPs.

We also studied the relationship between the number of RPs and the localisation accuracy of different models. Figure 2(b) shows that the model with a larger number of RPs achieves a better performance than with a smaller number of RPs. When the number of RPs is 60 , GM has reached $94.6 \%$ of the localisation accuracy of the original radio map. This means GM can use only a small amount of resource in updating the radio map.

4.1.3. Evaluation of KMGPR algorithm. To evaluate the performance of the KMGPR algorithm, we also conducted experiments in Scenario 1. In our experiment, we set the number of clusters to $1 \sim 6$ and randomly chose the clusters' centroid points. The aim was to identify the efficiency of the KMGPR algorithm when compared to GPR.

We used the Gaussian mean function-based GPR prior model to update the radio map. During the experiments, 10-fold cross-validation was performed for GPR and KMGPR. When KMGPR took different clusters with K-Means, the randomly selected RPs were different. In order to keep experimental consistency, GPR applied the same selected RPs as KMGPR. So, the results of GPR were different when KMGPR took different numbers of clusters.

Figure 3(a) depicts the average prediction error of different clusters in Scenario 1. Remarkably, when the number of clusters is 1, the KMGPR algorithm will become the GPR algorithm. The prediction accuracy of the KMGPR algorithm reached about $95 \%$ of the prediction accuracy of the GPR model, but the KMGPR algorithm has good performance in computational complexity as shown in Figure 3(b).

Figure 3(b) shows the time for updating the radio map with different numbers of clusters in Scenario 1. The results show that the KMGPR algorithm can cut the computation by about $16 \% \sim 24.1 \%$ when compared with GPR.

\subsection{Experiments.}

4.2.1. Experimental setup. To further evaluate our proposed KMGPR algorithm, an experiment was conducted on the third floor of No. 13 office building in Shandong University of Science and Technology (Figure 4). The floor is approximately $70 \mathrm{~m}$ by $50 \mathrm{~m}$ in size and six APs provide full coverage throughout the building. We developed software for a mobile device (Android 4.4) to collect the RSSI and test data. Fingerprint collections were taken at 65 reference points marked by white dots. For each reference point, we collected 20 RSS samples from each AP and then obtained averages of the results. There was about 
(a)

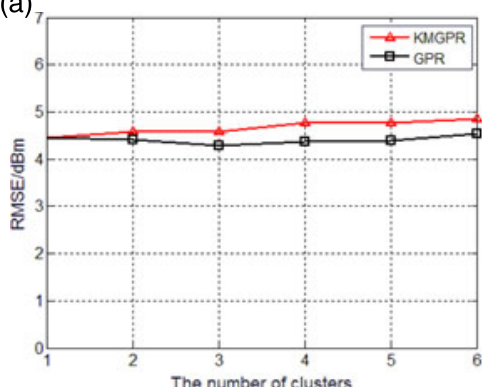

(b)

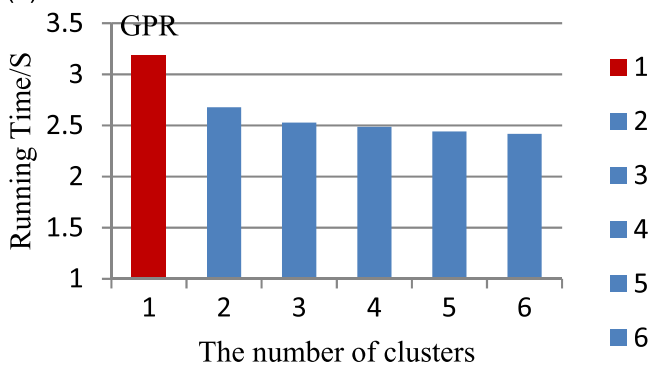

Figure 3. RMSE of prediction and running time with varying clusters. (a) RMSE of prediction; (b) Running time.

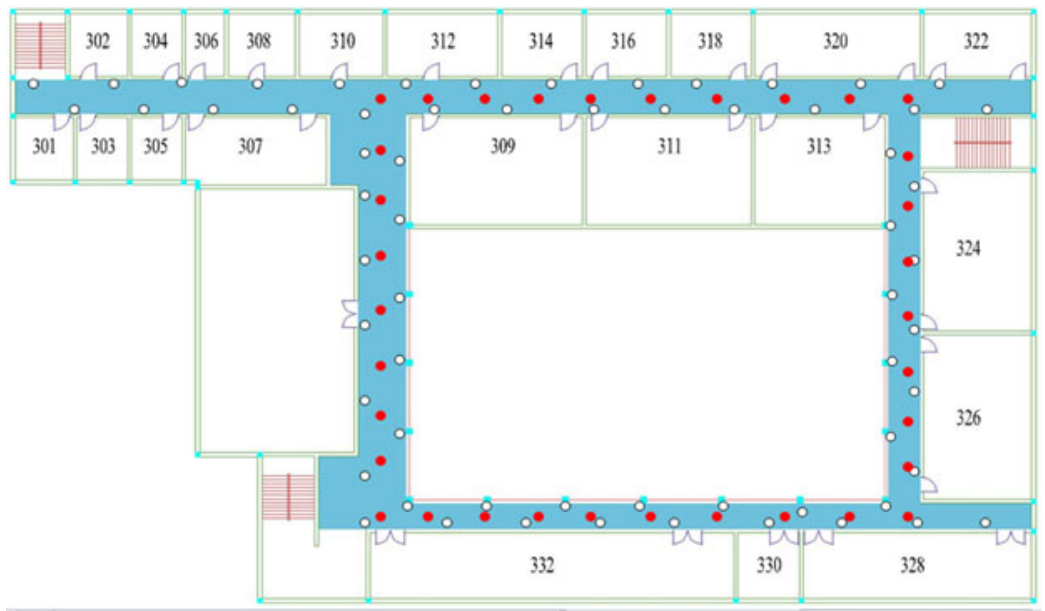

Figure 4. Experimental area (Scenario 2)

2 seconds between consecutive samples. This method can solve the problem of signal fluctuation. We collected other RSS samples at 34 locations marked by red dots as localisation test data. We collected the test data by walking along a predefined path in the corridor, which did not coincide with the reference points. This data can be used to evaluate our algorithm in conjunction with the training data. Figure 5 shows the locations of the real experimental data collection for Scenario 2.

4.2.2. GPR model evaluation. In Figure 4, there are 65 reference points in the positioning area. Different numbers of reference points were randomly selected for this experiment: $15,30,45$ and 60 . To illustrate the quality of GM in updating the RSS radio map, we also compared three mean functions: Zero, Const and Lin.

4.2.2.1. RSS estimation accuracy. In the experiment we used 10-fold cross-validation to determine the quality of the models and the test error and the RMSE of different models were investigated.

Table 5 shows the test error and RMSE of the GPR model for different mean functions. The analysis of the test error and RMSE showed similar experimental results to Scenario 1. 


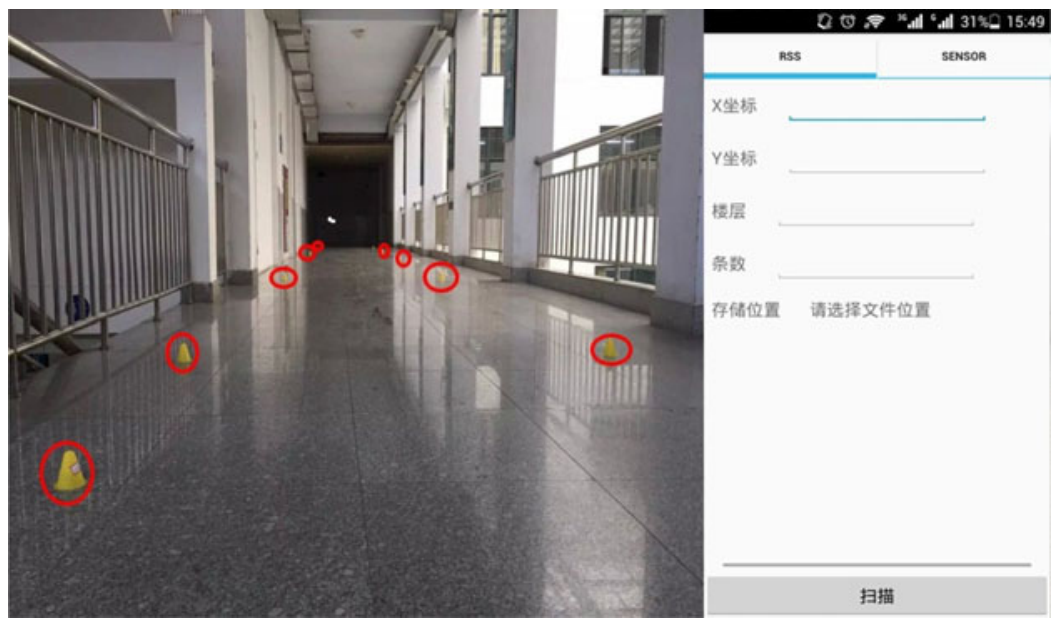

Figure 5. Fingerprint collection in Scenario 2.

Table 5. Test error and RMSE of the GPR model for different mean functions.

\begin{tabular}{|c|c|c|c|c|c|c|c|c|}
\hline \multirow{2}{*}{$\begin{array}{l}\text { Mean Function/ } \\
\text { The number } \\
\text { of RPs }\end{array}$} & \multicolumn{4}{|c|}{ Test Error/dBm } & \multicolumn{4}{|c|}{$\mathrm{RMSE} / \mathrm{dBm}$} \\
\hline & Zero & Const & Lin & GM & Zero & Const & Lin & GM \\
\hline 15 & $25 \cdot 42$ & $11 \cdot 37$ & $16 \cdot 45$ & $7 \cdot 306$ & $29 \cdot 25$ & $12 \cdot 25$ & $18 \cdot 04$ & $7 \cdot 389$ \\
\hline 30 & 5.087 & 4.86 & $6 \cdot 129$ & $4 \cdot 324$ & 5.624 & 4.921 & $6 \cdot 303$ & 4.401 \\
\hline 45 & $4 \cdot 243$ & 3.97 & $6 \cdot 035$ & $3 \cdot 885$ & 4.428 & $4 \cdot 136$ & $6 \cdot 226$ & 3.982 \\
\hline 60 & $3 \cdot 317$ & $3 \cdot 24$ & $5 \cdot 39$ & 2.953 & $3 \cdot 257$ & $3 \cdot 395$ & $5 \cdot 662$ & 2.989 \\
\hline
\end{tabular}

Table 6. Average localisation error for different mean functions.

\begin{tabular}{lcccc}
\hline \multirow{2}{*}{$\begin{array}{l}\text { The number } \\
\text { of RPs }\end{array}$} & \multicolumn{4}{c}{ Localisation Error/m } \\
\cline { 2 - 5 } & Zero & Const & Lin & GM \\
\hline 15 & 4.389 & 3.506 & 3.424 & 3.174 \\
30 & 2.812 & 2.608 & 2.979 & 2.407 \\
45 & 2.424 & 2.401 & 2.419 & 2.388 \\
60 & 2.381 & 2.264 & 2.915 & 1.984
\end{tabular}

GM can give a better result and consistently outperformed Zero, Const and Lin. Compared with other mean functions, the test error and RMSE of GM were $2.14 \sim 71.26 \%$ and $8.23 \sim 74.74 \%$ lower, respectively, in Scenario 2.

4.2.2.2. Localisation accuracy estimation. In this test, the 34 test points of Scenario 2 were used for localisation to verify the performance of GM. Localisation error is listed in Table 6 using the WKNN algorithm. For different numbers of training data sets, the most accurate GPR model is the GM model. When compared with other models, the localisation accuracy of GM can be improved by an average of $12 \cdot 50 \%$.

In Figure 6(a), the CDF of the localisation error for different mean functions is shown. ORI is the original radio map from all the reference points in Scenario 2. The result is consistent with Scenario 1. Figure 6(b) shows the CDF of localisation error for different 
(a)

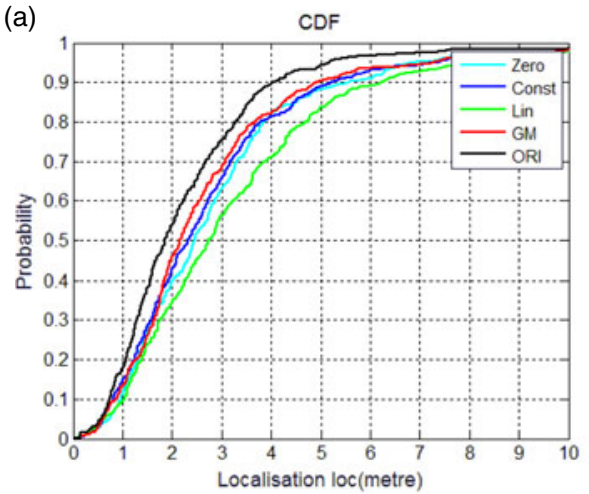

(b)

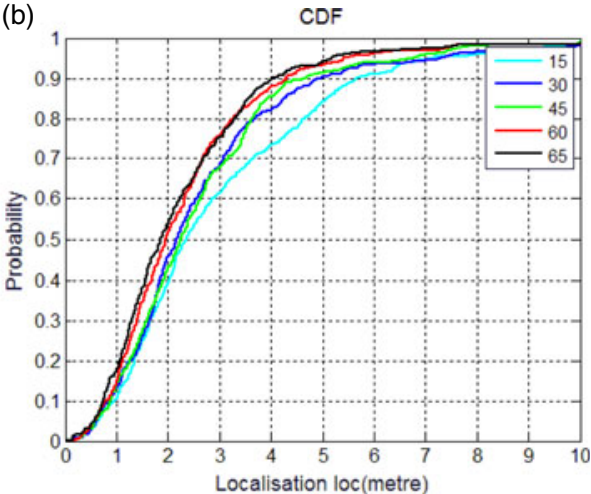

Figure 6. CDF of localisation error. (a) different mean functions; (b) different numbers of RPs.

(a)
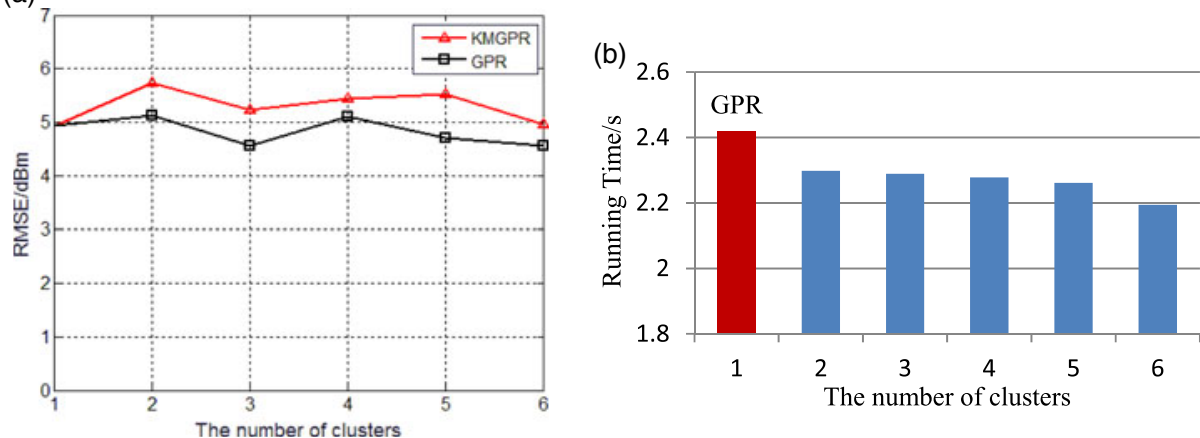

Figure 7. RMSE of prediction and running time with varying clusters. (a) RMSE of prediction; (b) Running time.

numbers of RPs. When the number of RPs is 60, GM has almost reached the localisation accuracy of the original radio map.

4.2.3. KMGPR in Scenario 2. Figure 7(a) shows the average prediction error for GPR and KMGPR with different clusters. Compared to the GPR model, the prediction accuracy of the KMGPR algorithm is only slightly reduced.

Figure 7(b) depicts the running time for updating the radio map with different numbers of clusters in Scenario 2. It shows that the time decreases when the number of clusters increases. The efficiency of our KMGPR algorithm increased by about $5 \sim 9.3 \%$ compared with GPR.

Figure 8 shows the localisation error versus the number of clusters for GPR and KMGPR in Scenario 2. The localisation errors between GPR and KMGPR are in a small range of fluctuations. As the number of clusters increases, the localisation error decreases first and then increases for KMGPR. This is because with the increase of the number of clusters, some potentially useful information contained in other RPs is lost. In summary, the KMGPR algorithm can have a good localisation performance with less workload compared to GPR. 


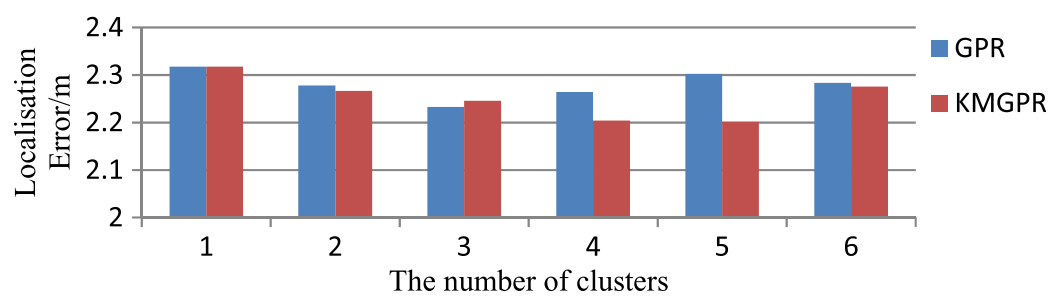

Figure 8. Localisation error comparison between GPR and KMGPR.

Thus, we can see that the KMGPR performs better. The KMGPR algorithm can improve the operating efficiency of online radio map updating and reduce system overhead without sacrificing localisation accuracy.

5. CONCLUSION. In this paper, an efficient algorithm for radio map updating based on K-Means and Gaussian Process Regression is introduced to reduce the workload in updating a fingerprint-based indoor localisation radio map. We first introduce the theory of GPR, parameter optimisation and clustering technology under WLAN-based indoor localisation. Then we build a GPR predictive model based on a Gaussian mean function and update the radio map with online K-Means clustering. Simulations and experiments have been conducted for the proposed KMGPR method and the results show that GPR using Gaussian mean function improves localisation accuracy by about $13.76 \%$ compared with other functions and KMGPR can reduce the computational complexity by about $7 \%$ and $20 \%$, respectively, in the two scenarios we investigated, with no obvious effects on the localisation accuracy.

\section{ACKNOWLEDGMENTS}

This paper is supported by National Key R\&D Plan (NO. 2017YFC0804406), Key R\&D Plan of Shandong Province (GG201709160017), Project of Industrial Transformation and Upgrading (Made in China 2025 NO.TC170A5SW) and Open Research Fund Program of Beijing Key Laboratory on Integration and Analysis of Large-scale Stream Data (NO.KF201802).

\section{REFERENCES}

Atia, M.M., Noureldin, A. and Korenberg, M.J. (2013). Dynamic online-calibrated radio maps for indoor positioning in wireless local area networks. IEEE Transactions on Mobile Computing, 12(9), 1774-1787.

Au, A.W.S., Feng, C., Valaee, S., Reyes, S., Sorour, S., Markowitz, S.N., Gold, D., Gordon, K. and Eizenman, M. (2013). Indoor tracking and navigation using received signal strength and compressive sensing on a mobile device. IEEE Transactions on Mobile Computing, 12(10), 2050-2062.

Bekkali, A., Masuo, T., Tominaga, T., Nakamoto, N. and Ban, H. (2011). Gaussian processes for learning-based indoor localization. Signal Processing, Communications and Computing (ICSPCC), 2011 IEEE International Conference on, 1-6.

Chai, X. and Yang, Q. (2007). Reducing the calibration effort for probabilistic indoor location estimation. IEEE Transactions on Mobile Computing, 6(6), 649-662.

Chang, K. and Han, D. (2014). Crowdsourcing-based radio map update automation for Wi-Fi positioning systems. Proceedings of the 3rd ACM SIGSPATIAL International Workshop on Crowdsourced and Volunteered Geographic Information, 24-31.

Chintalapudi, K., Padmanabha Iyer, A. and Padmanabhan, V.N. (2010). Indoor localization without the pain. Proceedings of the sixteenth annual international conference on Mobile computing and networking, 173-184. 
Ferris, B., Fox, D. and Lawrence, N. (2007). WiFi-SLAM using Gaussian process latent variable models. International Joint Conference on Artifical Intelligence (2480-2485). Morgan Kaufmann Publishers Inc.

Ferris, B., Hähnel, D. and Fox, D. (2006). Gaussian processes for signal strength-based location estimation. Proceedings of Robotics Science and Systems.

Hansen, R., Wind, R., Jensen, C.S. and Thomsen, B. (2010). Algorithmic strategies for adapting to environmental changes in 802.11 location fingerprinting. Indoor Positioning and Indoor Navigation (IPIN), 2010 International Conference on, 1-10.

Ji, S., Ma, H., Liang, Y., Leung, H. and Zhang, C. (2017). A whitelist and blacklist-based co-evolutionary strategy for defensing against multifarious trust attacks. Applied Intelligence 47(4), 1115-1131.

Jun, J., Gu, Y., Cheng, L., Lu, B., Sun, J., Zhu, T. and Niu, J. (2013). Social-Loc: Improving indoor localization with social sensing. Proceedings of the 11th ACM Conference on Embedded Networked Sensor Systems, 14.

Kaemarungsi, K. and Krishnamurthy, P. (2012). Analysis of WLAN's received signal strength indication for indoor location fingerprinting. Pervasive and Mobile Computing, 8(2), 292-316.

Kim, Y., Chon, Y. and Cha, H. (2012). Smartphone-based collaborative and autonomous radio fingerprinting. IEEE Transactions on Systems, Man, and Cybernetics, Part C (Applications and Reviews), 42(1), 112-122.

Kumar, S., Hegde, R.M. and Trigoni, N. (2016). Gaussian Process Regression for Fingerprinting based Localization. Ad Hoc Networks, 51, 1-10.

Laoudias, C., Piché, R. and Panayiotou, C.G. (2013). Device self-calibration in location systems using signal strength histograms. Journal of Location Based Services, 7(3), 165-181.

Liu, K., Meng, Z. and Own, C.M. (2016). Gaussian Process Regression Plus Method for Localization Reliability Improvement. Sensors, 16(8), 1193.

Mahtab Hossain, A.K.M., Jin, Y., Soh, W.S. and Van, H.N. (2013). SSD: A robust RF location fingerprint addressing mobile devices' heterogeneity. IEEE Transactions on Mobile Computing, 12(1), 65-77.

Mihaylova, L., Angelova, D., Bull, D. and Canagarajah, N. (2011). Localization of mobile nodes in wireless networks with correlated in time measurement noise. IEEE Transactions on Mobile Computing, 10(1), 44-53.

Pan, S.J., Kwok, J.T., Yang, Q. and Pan, J.J. (2007). Adaptive localization in a dynamic WiFi environment through multi-view learning. National Conference on Artificial Intelligence (Vol.2, 1108-1113). AAAI Press.

Park, J.G., Charrow, B., Curtis, D., Battat, J., Minkov, E., Hicks, J., Teller, S. and Ledlie, J. (2010). Growing an organic indoor location system. International Conference on Mobile Systems, Applications, and Services (271-284). DBLP.

Rai, A., Chintalapudi, K.K., Padmanabhan, V.N. and Sen, R. (2012). Zee: zero-effort crowdsourcing for indoor localization. Proceedings of the 18th Annual International Conference on Mobile Computing and Networking, 293-304

Rasmussen, C.E. and Williams, C.K.I. (2005). Gaussian Processes for Machine Learning (Adaptive Computation and Machine Learning). The MIT Press.

Richter, P. and Toledano-Ayala, M. (2015). Revisiting Gaussian Process Regression Modeling for Localization in Wireless Sensor Networks. Sensors, 15(9), 22587-22615.

Seitz, J., Jahn, J., Boronat, J.G., Vaupel, T., Meyer, S. and Thielecke, J. (2010). A hidden Markov model for urban navigation based on fingerprinting and pedestrian dead reckoning. Information Fusion (FUSION), 2010 13th Conference on, 1-8.

Wu, C., Yang, Z., Liu, Y. and Xi, W. (2013). WILL: Wireless indoor localization without site survey. IEEE Transactions on Parallel and Distributed Systems, 24(4), 839-848.

Wu, C., Yang, Z. and Liu, Y. (2015a). Smartphones based crowdsourcing for indoor localization. IEEE Transactions on Mobile Computing, 14(2), 444457.

Wu, C., Yang, Z., Xiao, C., Yang, C., Liu, Y. and Liu, M. (2015b). Static power of mobile devices: Selfupdating radio maps for wireless indoor localization. 2015 IEEE Conference on Computer Communications (INFOCOM), 2497-2505.

Wu, W., Zhao, J., Zhang, C., Meng, F., Zhang, Z., Zhang, Y. and Sun, Q. (2017). Improving performance of tensor-based context-aware recommenders using bias tensor factorization with context feature auto-encoding. Knowledge-Based Systems, 128(C), 71-77.

Yang, S., Dessai, P., Verma, M. and Gerla, M. (2013). FreeLoc: Calibration-free crowdsourced indoor localization. INFOCOM, 2013 Proceedings IEEE, 2481-2489.

Yu, K. and Dutkiewicz, E. (2013). NLOS identification and mitigation for mobile tracking. IEEE Transactions on Aerospace and Electronic Systems, 49(3), 1438-1452. 